\title{
ONE-DIMENSIONAL DYNAMICS: THE SCHWARZIAN DERIVATIVE AND BEYOND
}

\author{
W. DE MELO AND S. VAN STRIEN
}

Most of the important results in the study of the dynamics of smooth interval maps $f:[0,1] \rightarrow[0,1]$ assume the condition that $S f<0$ where $S f$ is the Schwarzian derivative of $f$ :

$$
S f=\frac{D^{3} f}{D f}-\frac{3}{2}\left(\frac{D^{2} f}{D f}\right)^{2} .
$$

This condition, although very powerful, has the disadvantage of being too restrictive and, even worse, it is not invariant under $C^{\infty}$ change of coordinates. More precisely, there exists a $C^{\infty}$ diffeomorphism $\varphi:[0,1] \rightarrow[0,1]$ such that $\varphi f \varphi^{-1}$ does not have negative Schwarzian derivative.

In this announcement we will present a technique which enables one to replace these conditions by smoothness conditions: we assume that $f$ is $C^{3}$ and that $f$ is nonflat at the critical points (i.e. $f$ is $C^{\infty}$ near the critical points and at each critical point one of the derivatives is nonzero). We will illustrate this technique by showing the analogue, for maps $f:[0,1] \rightarrow[0,1]$ with one critical point, of the result of Denjoy done for $C^{2}$ circle-diffeomorphisms.

More precisely, Denjoy showed that a $C^{2}$ diffeomorphism $f: S^{1} \rightarrow S^{1}$ cannot have any wandering interval $L \subset S^{1}$. Here, we say that $L$ is a wandering interval if $L, f(L), f^{2}(L), \ldots$ are mutually disjoint and no point $x \in L$ is asymptotic to a periodic orbit. From this it follows that if $f$ is a $C^{2}$ diffeomorphism, then either $f$ has a periodic orbit or it is conjugate to a rigid rotation. We say that $f:[0,1] \rightarrow[0,1]$ is in class $A$ if $f$ is a $C^{3}$ map with only one critical point and $f$ is nonflat at its critical point.

THEOREM. Let $f:[0,1] \rightarrow[0,1]$ be in class $A$. Then $f$ has no wandering intervals.

COROLLARY. Every $f$ in $A$ is semiconjugate to a map from the quadratic family $f_{\lambda}:[0,1] \rightarrow[0,1]$ defined by $f_{\lambda}(x)=\lambda x(1-x)$. This semiconjugacy only collapses the basin of attraction of the periodic orbits which do not attract the critical point.

REMARK 1. The Schwarzian derivative was introduced in one-dimensional dynamics by D. Singer [S]. Guckenheimer proved the nonexistence of wandering intervals for maps in $A$ under the assumption that $S f<0[\mathbf{G}]$.

REMARK 2. In general a map $f$ in $A$ can have several attracting periodic orbits, whereas if $f_{\lambda}$ has an attracting periodic point then it attracts the

Received by the editors November 1, 1986 and, in revised form, October 19, 1987.

1980 Mathematics Subject Classification (1985 Revision). Primary 58F13; Secondary $54 \mathrm{H} 20$. 
critical point $\frac{1}{2}$. It follows that one cannot hope to get a conjugacy between $f$ and $f_{\lambda}$.

REMARK 3. We expect to be able to prove that there is a bound for the period of the attracting periodic orbits of each map $f$ in $A$. This would imply that the semiconjugacy only collapses a finite number of intervals and their backward orbits.

SKETCH OF THE PROOF. We consider two cross-ratios. Let $J, T \subset[0,1]$ be open intervals such that $\operatorname{Clos}(T)-J$ has two connected components $L$ and $R$. We define

$$
C(T, J)=\frac{|J||T|}{|L \cup J||J \cup R|} \quad \text { and } \quad D(T, J)=\frac{|J||T|}{|L||R|},
$$

where $|J|$ denotes the length of the interval $J$. If $g:[0,1] \rightarrow[0,1]$ is monotone on $T$ we define the operators

$$
A(g, T, J)=\frac{C(g(T), g(J))}{C(T, J)} \quad \text { and } \quad B(g, T, J)=\frac{D(g(T), g(J)}{D(T, J)}
$$

If $g$ has negative Schwarzian derivative we can see that $A(g, T, J)>1$ and $B(g, T, J)>1$. In the general case we prove the following:

THEOREM 1. Let $f:[0,1] \rightarrow[0,1]$ be a $C^{\infty}$ map whose critical points are nonflat. There exist $\delta>0$ and $\frac{1}{18}>\epsilon>0$ such that if $T \supset J$ are open intervals satisfying: (i) $f^{m}$ is a diffeomorphism on $\operatorname{Clos}(T)$; (ii) $\sum_{k=0}^{\infty}\left|f^{k}(J)\right|<\delta$; (iii) $|L||R|<\epsilon|J|^{2}$ then

$$
A\left(f^{m}, T, J\right)<1-\frac{8|L||R|}{|J|^{2}}
$$

COROLLARY. Under the conditions of Theorem 1 we have

$$
\frac{\left|f^{m}(L)\right|\left|f^{m}(R)\right|}{|L||R|}<\frac{18}{|J|^{2}}\left|f^{m}(J)\right|\left|f^{m}(T)\right| \text {. }
$$

THEOREM 2. Let $f:[0,1] \rightarrow[0,1]$ be a $C^{\infty}$ map whose critical points are nonflat. There exists a constant $C_{1}>0$ such that if $T \supset J$ are intervals such that (i) $f^{m}$ is a diffeomorphism on $\operatorname{Clos}(T)$; (ii) $\sum_{k=0}^{m}\left|f^{m}(T)\right|^{2}=S<3$, then

$$
\log B\left(f^{m}, T, J\right)>-C_{1} S \text {. }
$$

THEOREM 3. Let $f:[0,1] \rightarrow[0,1]$ be a $C^{\infty}$ map whose critical points are nonflat. Let $C_{1}$ be as in Theorem 2. If $T=[a, b] \subset[0,1]$ is such that $f^{m}$ is a diffeomorphism on $T$ and $\sum_{i=0}^{m-1}\left|f^{i}(T)\right|=\delta<1$ then

$$
\left|D f^{m}(x)\right| \geq\left(\operatorname{Exp}\left(-C_{1} S\right)\right)^{3}\left|D f^{m}(a)\right|
$$

or

$$
\left|D f^{m}(x)\right| \geq\left(\operatorname{Exp}\left(-C_{1} S\right)\right)^{3}\left|D f^{m}(b)\right|
$$

or both.

Suppose, by contradiction, that $f$ has a wandering interval $J$. By replacing $J$ by some iterate we may assume that $\sum_{k=0}^{\infty}\left|f^{k}(J)\right|<\delta$ and $f^{n}(\operatorname{Clos}(J))$ does not contain the critical point $c$ for every $n$. By the theorem of Schwartz 
[CE, pp. 111], the forward iterates of $J$ must accumulate at the critical point $c$. Hence we may define a sequence of integers $k(n)$ by $k(0)=0$ and $k(n)=$ $\min \left\{k ; f^{k(n-1)}(J) \supset\left\langle f^{k(n-1)}(J),\left(f^{k(n-1)}(J)\right)^{\prime}\right\rangle\right\}$. Here, for an interval $T$ which does not contain the critical point, $T^{\prime}$ denotes the interval $f^{-1}(f(T))-T$ and $\left\langle T, T^{\prime}\right\rangle$ is the smallest interval containing $T \cup T^{\prime}$. Let $V_{n}=\left\{x ; f^{n}(x) \in\right.$ $\operatorname{int}\left(\left\langle x, x^{\prime}\right\rangle\right)$ and $f^{i}(x) \notin\left\langle x, x^{\prime}\right\rangle$ for $\left.i<n\right\}$. As in $[\mathbf{G}]$, the image of the boundary points of each connected component of $V_{n}$ are fixed points of $f^{n}$. Furthermore, the first $n-1$ iterates of such a connected component are disjoint intervals. Using these facts for the connected component of $V_{k(n+1)-k(n)}$ containing $f^{k(n)}(J)$, and Theorem 3 , we get that there is a constant $e>0$ independent of $n$ such that

$$
\left|f^{k(n+1)}(J)\right|>e\left|f^{k(n)}(J)\right| \text {. }
$$

Let $K_{n}$ be the largest interval containing $J$ on which $f^{n}$ is monotone. Since $J$ is a wandering interval we have that $K_{n}-J=L_{n} \cup R_{n}$, where $R_{n}$ and $L_{n}$ are nonempty intervals whose lengths go to zero as $n$ goes to infinity. As in [G], we get that $f^{k(n)}\left(K_{k(n)}\right)$ contains either $f^{k(n-1)}(J)$ or $\left(f^{k(n-1)}(J)\right)^{\prime}$ and it contains also either $f^{k(n+1)}(J)$ or $\left(f^{k(n+1)}(J)\right)^{\prime}$. Hence, by interchanging $L_{k(n)}$ with $R_{k(n)}$ if necessary, we get

$$
\left|f^{k(n)}\left(L_{k(n)}\right)\right|>\alpha\left|f^{k(n-1)}(J)\right|
$$

and

$$
\left|f^{k(n)}\left(R_{k(n)}\right)\right|>\alpha\left|f^{k(n+1)}(J)\right|>e \alpha\left|f^{k(n)}(J)\right|,
$$

where $\alpha=\inf |D f(x)| /\left|D f\left(x^{\prime}\right)\right|$. Since $\left|f^{k(n)}(J)\right| \rightarrow 0$ as $n \rightarrow \infty$ we may choose a subsequence $n(i) \rightarrow \infty$ such that $\left|f^{k(n(i))}(J)\right|>\left|f^{k(n(i-1))}(J)\right|$. From the corollary of Theorem 1 we get

$$
\begin{aligned}
& \frac{\left|f^{k(n(i))}\left(L_{k(n(i))}\right)\right|\left|f^{k(n(i))}\left(R_{k(n(i))}\right)\right|}{\left|L_{k(n(i))}\right|\left|R_{k(n(i))}\right|} \\
& \quad \leq \frac{18}{|J|^{2}}\left|f^{k(n(i))}(J)\right|\left\{\left(\left|f^{k(n(i))}\left(L_{k(n(i))}\right)\right|+\left|f^{k(n(i))}(J)\right|\right.\right. \\
& \left.+\left|f^{k(n(i))}\left(R_{k(n(i))}\right)\right|\right\} .
\end{aligned}
$$

By shrinking $K_{n(i)}$ we get that

$$
\begin{aligned}
& \frac{\left|f^{k(n(i))}\left(L_{i}^{*}\right)\right|\left|f^{k(n(i))}\left(R_{i}^{*}\right)\right|}{\left|L_{k(n(i))}\right|\left|R_{k(n(i))}\right|} \leq \frac{\left|f^{k(n(i))}\left(L_{i}^{*}\right)\right|\left|f^{k(n(i))}\left(R_{i}^{*}\right)\right|}{\left|L_{i}^{*}\right|\left|R_{i}^{*}\right|} \\
& \quad \leq \frac{18}{|J|^{2}}\left|f^{k(n(i))}(J)\right|\left\{\left|f^{k(n(i))}\left(L_{i}^{*}\right)\right|+\left|f^{k(n(i))}(J)\right|+\left|f^{k(n(i))}\left(R_{i}^{*}\right)\right|\right\}
\end{aligned}
$$

for every $K_{i}^{*}=L_{i}^{*} \cup J \cup R_{i}^{*} \subset K_{n(i)}$. Choose $L_{i}^{*}$ and $R_{i}^{*}$ so that

$$
\left|f^{k(n(i))}\left(L_{i}^{*}\right)\right|=\min \left\{\left|f^{k(n(i))}(J)\right|, \alpha\left|f^{k(n(i-1))}(J)\right|\right\}
$$

and

$$
\left|f^{k(n(i))}\left(R_{i}^{*}\right)\right|=\min \left\{\left|f^{k(n(i))}(J)\right|, e \alpha\left|f^{k(n(i))}(J)\right|\right\}
$$


Then

$$
\begin{gathered}
\frac{\left|f^{k(n(i))}\left(L_{i}^{*}\right)\right|\left|f^{k(n(i))}\left(R_{i}^{*}\right)\right|}{\left|L_{k(n(i))}\right|\left|R_{k(n(i))}\right|} \leq 3 \frac{18}{|J|^{2}}\left|f^{k(n(i))}(J)\right|^{2} \\
\frac{\left|f^{k(n(i))}(J)\right|}{\left|f^{k(n(i))}\left(R_{i}^{*}\right)\right|}=\max \left(1,(e \alpha)^{-1}\right)
\end{gathered}
$$

and

$$
\frac{\left|f^{k(n(i))}(J)\right|}{\left|f^{k(n(i))}\left(L_{i}^{*}\right)\right|} \leq \max \left(1, \alpha^{-1}\right)
$$

because $\left|f^{k(n(i-1))}(J)\right|>\left|f^{k(n(i))}(J)\right|$. Hence

$$
\frac{1}{\left|L_{k(n(i))}\right|\left|R_{k(n(i))}\right|} \leq 3 \frac{18}{|J|^{2}} \max \left(1,(e \alpha)^{-1}\right) \max \left(1, \alpha^{-1}\right)
$$

This is a contradiction because $\left|L_{k(n(i))}\right|$ and $\left|R_{k(n(i))}\right|$ go to zero as $n(i) \rightarrow \infty$.

\section{REFERENCES}

[C-E] P. Collet and J. Eckmann, Iterated maps of the interval as dynamical systems, Birkhäuser, 1980.

[D] A. Denjoy, Sur les courbes définié par les équations différentielles à la surface du tore, J. Math. Pures Appl. 11 (1932), ser. 9.

[G] J. Guckenheimer, Sensitive dependence on initial conditions for one-dimensional maps, Comm. Math. Phys. 70 (1979), 133-160.

[Si] D. Singer, Stable orbits and bifurcations of maps of the interval, SIAM J. Appl. Math. 35 (1978), 260.

instituto de Matematica Pura e Aplicada, 110 Jardim Botanico, Rio DE JANEIRO, BRAZIL

DELFT UNIVERSity OF TECHNOLOGy, DELFT, THE NetherLANDS 